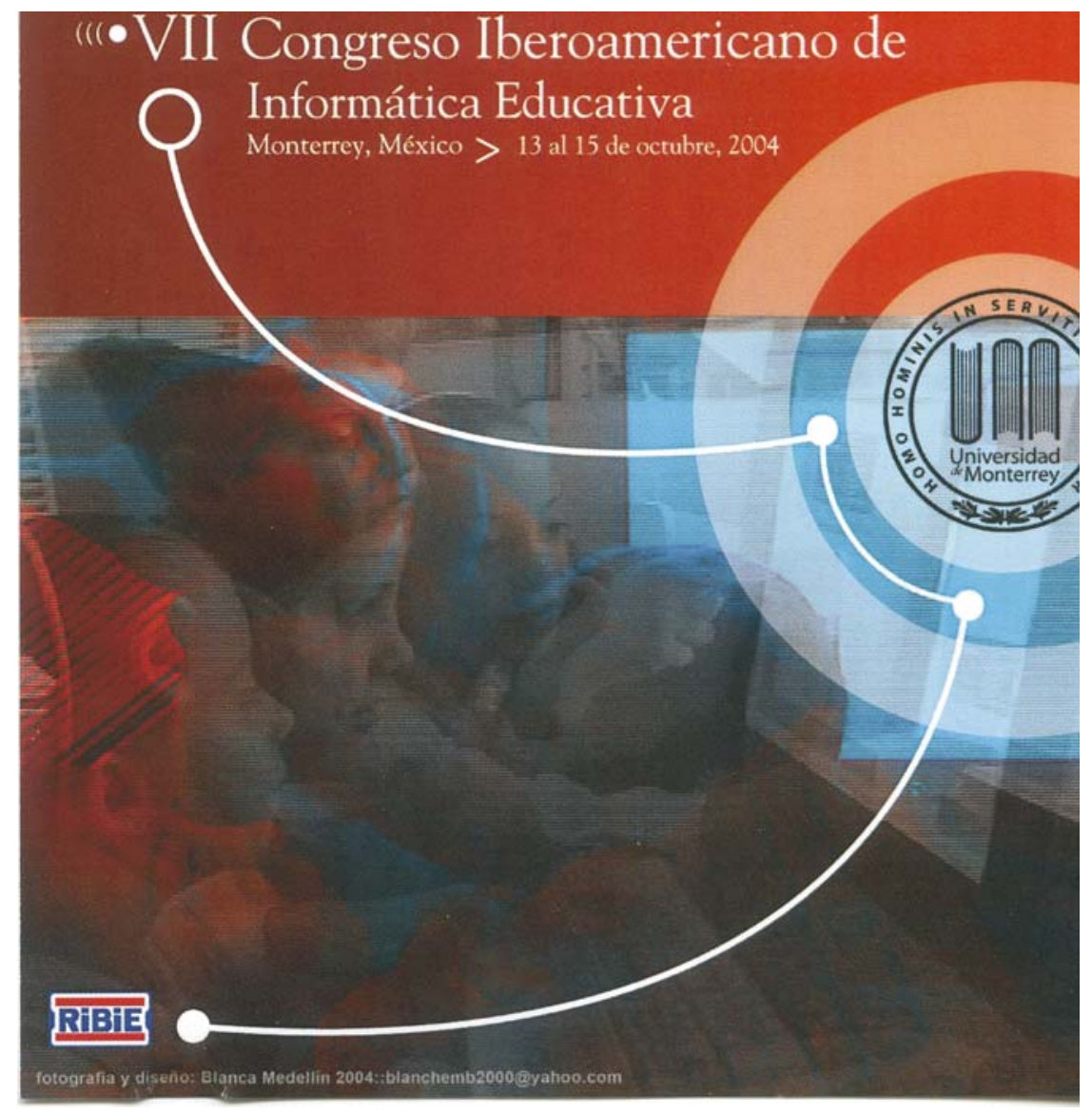




\title{
APL: AUDIO PROGRAMMING LANGUAGE FOR BLIND LEARNERS
}

\author{
Jaime Sánchez y Fernando Aguayo \\ Department of Computer Science \\ University of Chile \\ Blanco Encalada 2120, Santiago, CHILE \\ \{jsanchez, faguayo\}@dcc.uchile.cl
}

\begin{abstract}
Programming languages have been increasingly mapping end-users needs and mental models. They have expanded the number of users who can program or learn how to program. They are focused on sighted users. This study introduces APL, an Audio Programming Language for blind learners. APL is a programming language with audio-based interfaces to assist blind learners to develop problem solving and algorithmic thinking skills. APL is also a way to help blind learners to construct meaning by making programs. APL is a programming language by and for blind learners. We tested APL with novice blind programmers during and after development. They tried, analyzed and make improvements to APL. They usability tested APL by solving problems with increasingly complexity and answering usability questionnaires. Blind users understood APL, made programs, mapped the programming process and enjoyed the experience showing that a programming language based on audio can be constructed to fit the needs and mental models of blind learners to help them to enter to the programming world.
\end{abstract}

\section{Keywords}

Virtual acoustic environments, blind children, spatialized sound, audio-based navigation, usability

\section{INTRODUCTION}

In the last three decades diverse attempts have been made to make programming closer to end users: Basic, Logo, Smalltalk, Pascal, Boxer, Playground, KidSim, AgenSheets, LiveWord, Shoptalk $[4,6,11,12,17,18,20]$. All of these have contributed to expand the number of people who can program and learn how to program. Many of them have applied user interfaces principles to programming. This ends up with better skills to program by end users. Thus we can describe studies concerning Programming by Demonstration, Programming by Example, Visual Programming, Graphical programming, and Physical Programming $[2,3,5,6,8,10,13,21]$. Most of this programming attempts (if not all) has been focused on visual programmers.

A learner with visual disabilities that wants to learn programming has to use text-tospeech systems that "reads" programming commands, variables, etc. Actually, we can assume that there is an implicit assumption that blind users think in the same way as sighted users.

A number of recent studies have shown that when using audio-based applications blind children can develop and rehearse cognition [7, 9, 14, 15, 16, 19]. Most of these studies focus on the development of 3D audio interfaces to map the entire surrounding space as proof of 
concept concerning audio-based interfaces. A few studies analyses the effects and impact of spatialized sound on the development of cognition of blind people. They tend to evaluate the usability of these applications by solving cognitive tasks in order to develop tempo-spatial relationships, short and abstract memory, spatial abstraction, haptic perception, and mathematic reasoning.

This research study introduces APL, a programming language based on audio to enhance problem solving and thinking skills in novice blind learners. APL was built by and for blind learners. They usability tested different versions of the software and contribute to enrich and make APL to map their needs and way of thinking. Blind learners also evaluated the usability of the final version of APL by walking through it and solving specific tasks.

\section{A PROGRAMMING LANGUAGE FOR BLIND LEARNERS}

What are the specific needs of blind learners in order to program? Why is it difficult to them to map and follow current programming languages? Can we develop a programming language to better fit their needs? These were the underlying questions in our study.

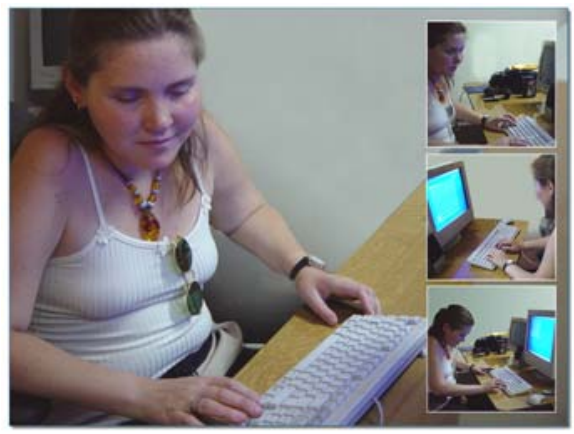

Figure 1. A blind learner interacting with APL by only using sound.

A programming language communicates the programmer with the computer. To do this the structure and logic is designed in such a way to be interpreted by the machine. Actual languages are based on the idea that a programmer writes command lines interpreted by the computer. These commands must be correctly written and well defined otherwise the machine cannot understand instructions and specific tasks are unsolved. This implies to memorize a huge amount of command lines and to write command lines correctly in order to avoid error parsing. These programming languages are heavily based on visual interfaces

Two major difficulties can be found when blind learners use these languages. First, if they use a pure language they will face the issue of verifying program consistency and the 
correct reading of command lines. Second, if we provide them with current tools to support program construction, they face the issue of dealing with graphical user interfaces. Actually, APL intends to close the gap between programming by sighted learners and programming by blind learners.

APL is the first intent to develop a programming language oriented to blind learners. The idea is that blind youth can interact and communicate with the computer as sighted programmers do by creating and manipulating their own programs and exchanging them with other users, sighted or blind. The only difference is the way the do it and the sensory channel used to interact with the machine.

APL has been conceived to make easier the writing of command lines by assuring correct writing and avoid parsing problems, and thus facilitating machine-programmer interaction through additional tools not commonly found in programming languages.

APL is targeted to a specific target users then it can be adapted to their specific needs. As an example, common programming languages use minimum storage units or variables that are appropriate to the user needs. Thus it is hard to image a language without string or integer variables. All emerge from the user's needs. In contrast, APL implements a new and unconventional type of variable that is able to store sounds to be later manipulated by the blind user. Sound is not a function of APL; rather it is treated as a fundamental unit.

\section{APL: AUDIO PROGRAMMING LANGUAGE}

We aimed at developing a tool to facilitate the manipulation of a programming language by blind learners. Then we provided facilitating elements oriented to blind novice programmers by diminishing syntax complexity of a current language and adding functions to analyze the final programs.

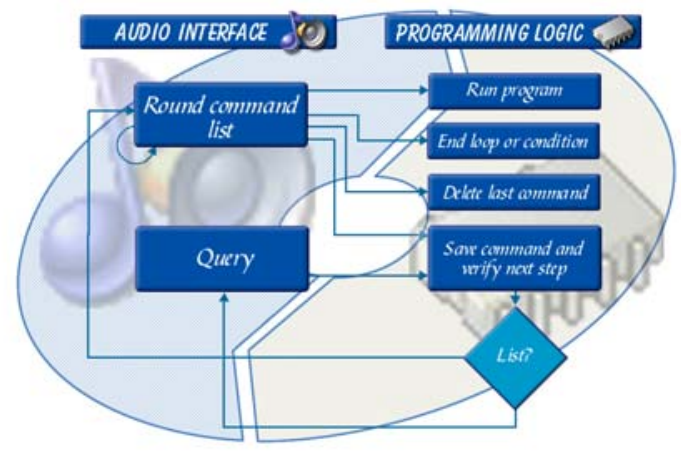

Figure 2. Layers of APL 


\section{Modeling}

We developed an implementation model. Two basic conditions were set. The software should be easy to implement and flexible by allowing changes without modifying everything. APL has two main layers: Audio Interface and Program Logic (see Figure 2).

Audio Interface includes two states: Round Command List and a Query. Round Command List contains a command list to be chosen according to the program state such as loop, condition, input, output, and variables. Query is used to define variable names and values, input/output audio. Text is optional.

Program Logic consist of four states: Run program, End loop or condition, Delete last command, and Save command and verify next step. All of them facilitate the correct semantic and make the logic understandable by the computer. The semantic is visible for the computer and invisible for the end user. Save command and verify next step controls actions through a self-questioning about if it is either a list or a query by triggering one of the Audio Interface states.

\section{Development}

APL was developed by using Java 1.4.1 and FreeTTS Java speech synthesizer. These are fine tools for media management and close to the machine logic. APL has the following modules: Data Base, Integrity, Kernel, and CHI.

Data Base stores the commands and its integrity. Integrity reads the Data Base loading on a certain structure and manipulating commands. APL has the following commands: loop, condition, input, output, and variables. Loop and condition have the same meaning as in current programming languages. Input and output are based on audio and text-to-speech defined by the blind programmer and saved as a variable for later manipulation. Variables are sound, text and number. Sound is the main and most used variable. Kernel manages the interaction between the user and programming rules. CHI controls input/output audio and translates input/output text to audio. The interface is entirely based on audio then blind programmers have not direct interaction with the screen. APL has two modes: programmer mode and running mode.

APL is a programming language for blind users. It favors information input and adds feedback adapted to users. To do this blind novice programmers should not write desired 
commands. Rather they can select them from a list classified by categories carefully designed to help searching expected commands. This assures a correct command semantic and helps to have an adequate parsing for the machine.

Blind programmers can choose commands within the following categories: loop, condition, input, output, cycle, and variables. They are the first category of commands and can be stored in a nested list thoroughly navigated by up and down arrows. APL reads each command from this list as demanded by the programmer. This is made through a text-tospeech embedded in APL. To move through the list of commands the programmer has to use up and down arrows to walk easily and completely throughout the rounded list. To choose a command the programmer has to select the right arrow. After entering the command a new list is downloaded underlying the preceding command. Thus a new valid command list is completed and stored in memory. Blind programmers repeat this process as many times as possible by selecting among a fixed number of commands to come out with the desired product. To execute the programmed application the user has to press F8.

The command definition is:

Input: The blind programmer uses this command to create an input requirement when executing the program by selecting the desired variable, keyboard or sound. This input should be saved in a variable defined by the programmer.

Output: It corresponds to the medium available to the programmer to create output of the product. Here we use only sound interfaces. Sound has two modes: 1. The programmer defines a variable and saving it with a corresponding sound, and 2 . The programmer defines a variable and saving it with a corresponding word or sentence entered through the keyboard and read by text-to-speech when executing the program.

Cycle: Command that allows executing the program many times in a defined sector. It can only come out of the defined sector when the defined cycle condition is attained. The cycle is completed by using the end cycle option.

Condition: Command that compares between defined variables by the programmer. If they are true the commands between tags are executed. Then a new option is created in the command list, end of condition. 
Variable: Here the variables are defined. They can be text or sound type. Text is read by textto-speech.

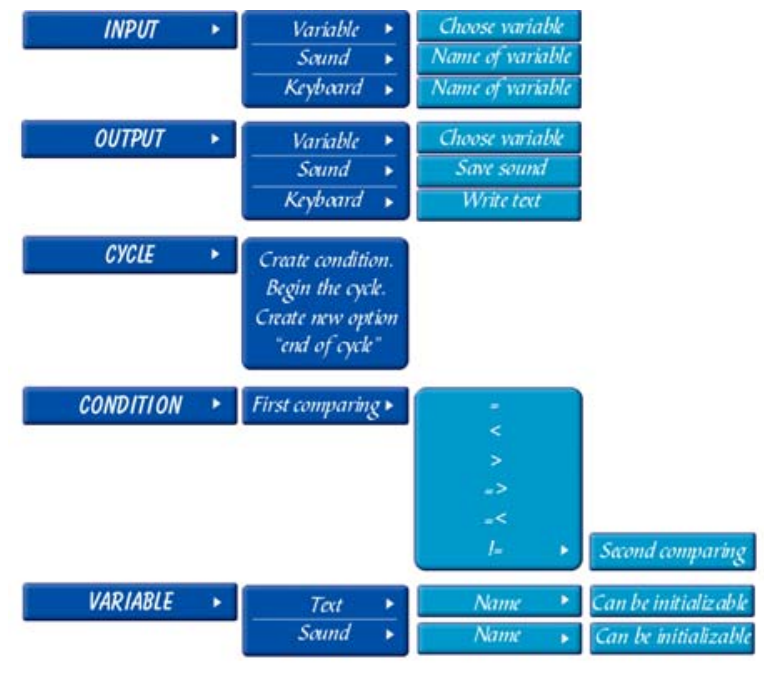

Figure 3. Command list tree

During the execution mode the programmed commands are interpreted and interact with the user and machine according to the requirements defined by the programmer. This module does not have a high interaction with the user because the main goal is to execute the program.

\section{PRELIMINARY USABILITY TESTING RESULTS}

The first prototype of APL has been tested with expert and end users.

Evaluation by expert users. APL was tested with four expert users. The main goal was to test APL with users that have experience and knowledge in programming to validate the functionality, to identify possible problems and errors, and to suggest some improvements. They were informed about the correspondence of APL commands with generic commands, and they programmed the software with the available commands. Experts detected functioning problems such as: a. APL did not support multiple cycles, b. APL did not allow to make waterfalls conditions, c. The conditions of APL did not allow to exit from cycles, d. Some issues when manipulating variables "keyboard" (variable types such int, var, and string cannot be defined), e. The sound and voice can be confusing in some aspects, f. The executor went 
down when the cycle was not appropriately defined. We then improved the prototype both the programmer interface and the execution by considering these comments and suggestions.

Evaluation by end users. APL was usability pilot tested with three blind novice programming learners, ages 17 to 20, during four long interactive sessions of 2.5 hours each. First, basic programming concepts were introduced and solved related mental tasks. Second, they interacted with APL by using basic commands to create simple programs such as Hello World and Jalisco (a guessing game). Third, they made their own programs such as "think an idea and make a program" (see Figure 1). Finally, they developed a small program for blind and sighted people to learn Braille.

The main goal was to familiarize users with concepts and elements to solve problems with APL. The idea was that users could interact with APL, identify classic algorithms and data structure in computer science, and develop algorithmic thinking to solve a problem. Users understood the functioning and the main goal of APL. They also learned about the basics of programming.

Blind users were observed by a team of three research people using a Likert type written scale and answered a usability test during the last session.

In the beginning they understood some programming concepts but it was complex to them to understand the concept and functions of different commands such as cycle, condition, and variable. This took more time as expected. During the third session they understood these concepts and made comments concerning APL based on their interaction experience and expectations about the functionality. We observed that methodologies such as theoretical explanations and step by step exercises did not help them to understand fully the basic concepts of programming.

This can be explained by knowing that blind learners tend to rely heavily on concrete experience before building abstract thinking. They did not construct a mental model of APL and programming until they had their own idea and knowing fully the meaning. Then from known to unknown they increasingly develop understanding of programming.

Blind learners were able to interact with APL by using commands such as input, output, variables, cycles, and condition. They could make some programs following step by step directions as well as making programs about ideas posed by them. By the last session they 
evidenced to understand programming concepts and apply them to their programs. Blind users constructed logic thinking skills, verbalized them with their partners and observers, and make programs to prove their feasibility. They were very motivated and satisfied as a result of interacting with APL.

As users understood concepts and commands, and applied them to their programming, they were able to design logic instructions, understand, reproduce and verbalize them. They also generated algorithms that can be reproduced mentally. They were motivated and showed interest and enthusiasm when programming.

They also made diverse comments and suggestions to improve APL such as to have a feedback by using the space bar to remind the last written word, to start up APL by their own, and to make it faster when using arrows.

Our experience with blind users programming APL shows that current programming visual tools are not feasible for these learners. It appears that the issue is not just to adapt visual programming to blind learners [18]. The theoretical background of these tools is complex to be mapped by novice blind learners. One interesting result in our usability test evidenced that interactivity may have rather different meaning and implications in blind users. This needs to be studied more fully.

\section{CONCLUSION}

We have introduced APL, a programming language based on audio for blind learners. We designed APL to help blind learners to enter to the programming world by targeting their needs and mental models. Blind learners were able to interact and program APL by using commands. They could understand programming concepts and apply them to their programs. Learners followed directions and programmed their own ideas.

Adequate uses of audio as a sensory interactive medium can help learners to learn how to program thus solving problems and developing thinking skills. We observed that throughout the testing sessions blind learners developed algorithmic and logic thinking skills, verbalized them, and wrote programs to prove their feasibility. The experience of interacting with APL was motivating and very interesting to them. When the testing concluded they expressed satisfaction as a consequence of this programming experience. 
We have learned the way blind users map the programming process by using APL. We believe it is somehow different from sighted users. Actually, the concept of interaction through programming has a different meaning to them. This is a first step in our attempt to provide a flexible and robust Audio Programming Language that fits the needs of blind learners and help them to enjoy learning to program and thus developing their cognition.

\section{ACKNOWLEDGMENTS}

This report was funded by the Chilean National Fund of Science and Technology, Fondecyt, Project 1030158.

\section{REFERENCES}

Baldis, J. Effects of spatial audio on memory, comprehension, and preference during desktop conferences. Proceeding of the ACM CHI '01, Vol 3, 1, (2001), pp. 166-173.

Cypher, A., Halbert, D. C., Kurlander, D., Lieberman, H., Maulsby, D., Mayers, B.A., and Turransky, A. (eds.). Watch What I Do: Programming by Demonstration. Cambridge, MA: The MIT Press, 1993

Cypher, A. and Smith, D. KidSim: End-user programming of simulations. In Proceedings of CHI'95. ACM. Denver, 1995.

Frenton, J. \& Beck, K. Playground: An object-oriented simulation system with agent rules for children of all ages. In Proceedings of OOPSLA '89. ACM, NewYork, 1989, pp. 123-137.

Ishii, H. and Ullmer, B. Tangible Bits: Toward Seamless Interfaces between People, Bits and Atoms. In Proceedings of CHI '97. ACM, Atlanta,GA, 1997, pp. 234-241.

Lieberman, H. Your Wish Is My Command: Programming by Example. Morgan Kaufmann, San Francisco, 2001.

McCrindle, R. \& Symons, D. Audio space invaders. Proceedings of the Third International Conference on Disability, Virtual Reality and Associated Technologies, (2000), pp. 59-65.

McDaniel, R. and Myers, B. Getting more out of Programming-By-Demonstration. In Proceedings of CHI '99. ACM, Pittsburgh PA, 1999, pp. 442-449.

Mereu, S. \& R. Kazman. Audio enhanced 3D interfaces for visually impaired users. Proceedings of CHI '96, ACM Press. (1996). 
Montemayor, J. Physical programming: software you can touch. In Proceedings of ACM SIGCHI, ACM Press, pp. 81-82, March 2001.

Nardi, B. A Small Matter of Programming Perspectives on End User Computing. MIT Press, Cambridge MA, 1993.

Repenning, A. AgentSheets: A tool for building domain-oriented dynamic, visual environments. Ph.D. Dissertation, Dept. of Computer Science, University of Colorado at Boulder, 1993.

Rosson, M. and Seals, C. Teachers as simulation programmers: minimalist learning and reuse. In Proceedings of CHI '01. ACM, Seattle WA, 2001, pp. 237-244.

Sánchez, J. Interactive 3D sound hyperstories for blind children. Proceedings of ACM CHI '99, (1999). Pittsburg PA, pp. 318-325.

Sánchez, J. Interactive virtual acoustic environments for blind children. Proceedings of ACM CHI '2001. Seattle Washington, April, (2001), pp. 23-2.

Sánchez, J. AudioBattleShip: Blind Learners Collaboration through Sound. Proceedings of ACM CHI' 03, (2003). Fort Lauderdale Florida, pp. 798-799.

Soloway, E., and Spohrer, J. Studying de Novice Programmer. Lawrence Erlbaum, Hillsdale, NJ, 1989.

Siegfried, R. A scripting language to help the blind to program visually. ACM SIGPLAN Notices, Vol 37 (2), pp.53-56, February 2002.

Sjostrom, C. Using haptics in computer interfaces for blind people. Proceeding of the ACM CHI '01, Vol 3, 1, (2001), pp. 245-246.

Travers, M. Recursive interfaces for reactive objects. In Proceedings of CHI '94. ACM, Boston, 1994, pp. 379-385.

Wilson, A., Brunett, M., Beckwith, L., Granatir, O., Casburn, L., Cook, C., Durham, M. and Rothermel, G. Harnessing curiosity to increase correctness in end-user programming. In Proceedings of CHI '03, Fort Lauderdale Florida, 2003, pp. 305-312 